\title{
Molecular dynamics simulation of Ion Beam Etching as technology process for creating graphene-based membranes
}

\author{
Łukasz Radosińskia, Piotr Żemojtel ${ }^{\mathrm{b}}$ \\ a Wrocław University of Science and Technology, Faculty of Chemistry, Department of Bioprocess and \\ Biomedical Engineering, Wrocław, Poland, lukasz.radosinski@pwr.edu.pl, +48713202838 \\ ${ }^{b}$ Wrocław University of Science and Technology, Faculty of Chemistry, Wrocław, Poland, czekodzemi@gmail.com
}

\begin{abstract}
Carbon nanomaterials present new possibilities for creating permeable membranes. Ionic beam etching technology allows to create pores in graphene substrates and has the potential to be used in various separation processes. In our study we present a molecular dynamics simulation of the energy needed to puncture the graphene surface to create porous surface. Experimental part shows dependency of irradiation time to pore diameter on silica substrate, which indicates it is possible to create pore diameters lesser than the beam diameter. We present a technology process of creating pores of a specific diameter.
\end{abstract}

Keywors - graphene, membrane, transport, ion beam, molecular dynamics.

\section{Introduction}

According to the definition, a membrane is a "structure, having lateral dimensions much greater than its thickness, through which mass transfer may occur under a variety of driving forces" [1]. Membranes processes can separate particles and molecules and over a wide particle size range and molecular weights. Efficiency in a membrane process is determined by a selectivity parameter. Unique structure of a one atom thick $\mathrm{sp}^{2}$-bonded carbon sheet of graphene presents unique properties than other forms of carbon in context to membranes. It has large theoretical specific surface area and even with pores its structure remains very resistant to pressure. It has potential for being a material to create very selective and permeable membranes, with personalized pore diameter for various processes e.g. gas separation, electrodialisys, nanofiltration [2]. Functionalizing its surface it further changes the properties of a porous membrane created from graphene sheet. As shown in the MIT computational study, transport of water through nano-porous graphene-oxide membranes could reach up to $66 \mathrm{~L} / \mathrm{cm} 2 / \mathrm{day} / \mathrm{MPa}$ with greater than $99 \%$ salt rejection. [3]. Creating a porous surface on a graphene can be carried out by focused ionic beam etching and it is possible to influence the pore diameter with respect to irradiation time and the by choosing an optimum angle [4].

In our work we use classical molecular dynamics code with a focus on materials modeling to develop a technology for creating a technological process for creating porous surface on graphene. 
We present the process for creating reliable molecular dynamics model of graphene surface ion beam etching carried out for various pore diameters. In our approach the model of single and several graphene sheets are hit by a fullerene model to imitate the energy density of ion beam. We created a dependency graphs of kinetic energy to pore diameter using several different fullerenes models. To our simulation we used the AIREBO [5] force field potential, which is a good system for modeling carbon and/or hydrogen atom interactions. The deflection of fullerene model indicates that with optimal angle and low enough kinetic energy it is possible to create pores of smaller diameter than the ion beam diameter.
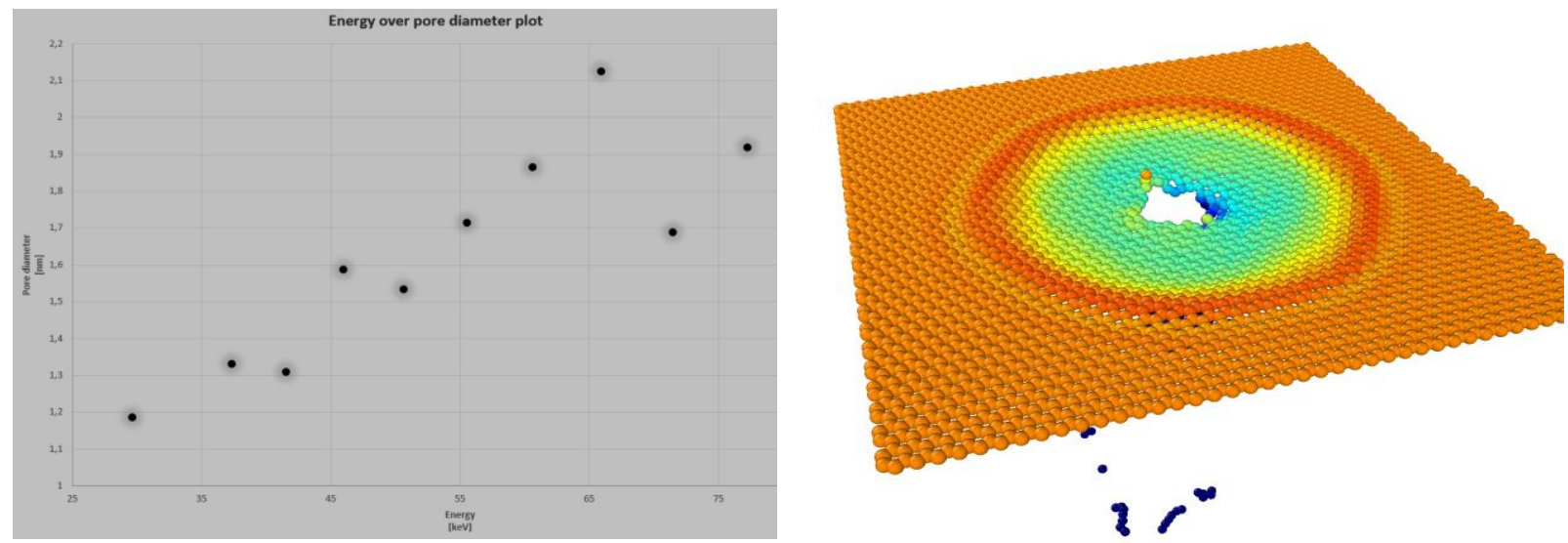

Figure 1. Left: Dependency of energy density to pore diameter in C180 fullerene model simulation, Right: Graphical representation for the impact simulation in molecular dynamics model created by LAMMPS package [6] with Ovito plugin [7]

Experimental part was to create a dependency of the pore diameter to the irradiation time. It was carried out with ionic beam etching on a silicon substrate.
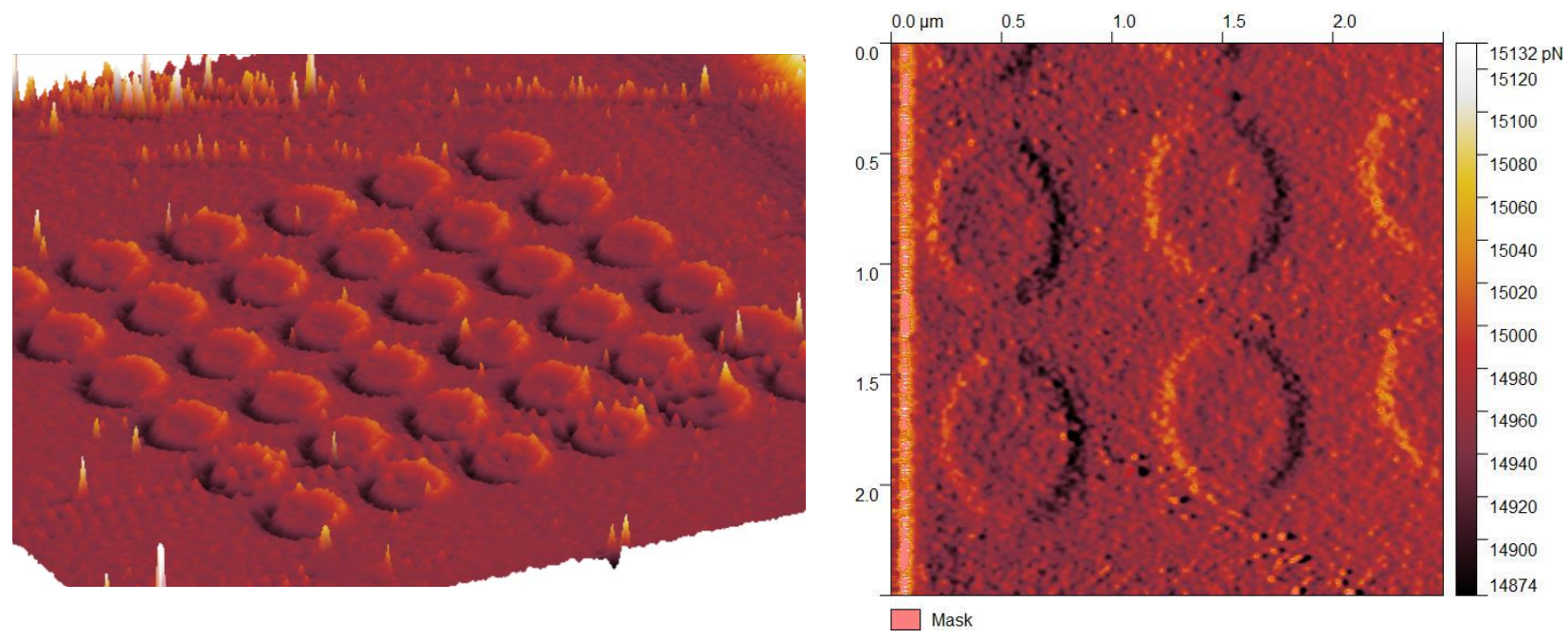
Figure 2. AFM pictures of Silicon substrate after FIB etching done in Nanores company in Wrocław.

\section{Conclusion}

The molecular dynamics model can very well be used to imitate the ion beam irradiation on the graphene surface. The characteristics of graphene's response to ion irradiation can be used to gain detailed control over produced defect types. This information is needed in order to controllably create porous membrane from this material, and then to create a experimental installation using this type of molecular sieve.

\section{References}

1. IUPAC. Compendium of Chemical Terminology, 2nd ed. (the "Gold Book"). Compiled by A. D. McNaught and A. Wilkinson. Blackwell Scientific Publications, Oxford (1997). XML on-line corrected version: http://goldbook.iupac.org (2006-) created by M. Nic, J. Jirat, B. Kosata; updates compiled by A. Jenkins. ISBN 0-9678550-9-8. https://doi.org/10.1351/goldbook.

2. R.K. Joshi, P. Carbone, F.C. Wang, V.G. Kravets, Y. Su, I.V. Grigorieva, H.A. Wu, A.K.Geim, R.R. Nair, Precise and Ultrafast Molecular Sieving Through Graphene Oxide Membranes, Science 343 (2014) 752-754.

3. Water Desalination across Nanoporous Graphene David Cohen-Tanugi and Jeffrey C. Grossman Nano Letters 201212 (7), 3602-3608

4. O. Lehtinen, J. Kotakoski, A. V. Krasheninnikov and J Keinonen Cutting and controlled modification of graphene with ion beams, Nanotechnology 22 175306, 2011

5. O'Connor, Thomas $\mathrm{C}$, Jan W. Andzelm and Mark O. Robbins. "AIREBO-M: a reactive model for hydrocarbons at extreme pressures." The Journal of chemical physics 1422 (2015): 024903 .

6. S. Plimpton, Fast Parallel Algorithms for Short-Range Molecular Dynamics, J Comp Phys, 117, 1-19 (1995). http://lammps.sandia.gov

7. A. Stukowski, Visualization and analysis of atomistic simulation data with OVITO - the Open Visualization Tool, Modelling Simul. Mater. Sci. Eng. 18 (2010), 015012 http://ovito.org/ 